CLINICAL STUDY

\title{
A unique exonic splicing mutation in the CYP17A1 gene as the cause for steroid $17 \alpha$-hydroxylase deficiency
}

\author{
Jie Qiao ${ }^{1, *}$, Bing $\mathrm{Han}^{1,2, *}$, Bing-Li Liu ${ }^{2, *}$, Wei Liu ${ }^{2}$, Jia-Jun $\mathrm{Wu}^{1}$, Chun-Ming $\mathrm{Pan}^{2}$, He Jiang ${ }^{2}$, Ting Gu ${ }^{1}$, \\ Bo-Ren Jiang ${ }^{1}$, Hui Zhu ${ }^{1}$, Ying-Li Lu ${ }^{1}$, Wan-Ling $\mathrm{Wu}^{1}$, Ming-Dao Chen ${ }^{2}$ and Huai-Dong Song ${ }^{2}$ \\ ${ }^{1}$ Department of Endocrinology, Shanghai Ninth People's Hospital, Shanghai Jiao Tong University School of Medicine, 369 Zhizaoju Road, Shanghai \\ 200011, People's Republic of China and ${ }^{2}$ State Key Laboratory of Medical Genomics, Shanghai Institute of Endocrinology, Molecular Medical Centre, \\ Ruijin Hospital, Shanghai Jiao Tong University School of Medicine, Shanghai 200025, People's Republic of China \\ (Correspondence should be addressed to Y-L Lu; Email: qiaoj2001@126.com)
}

*(J Qiao, B Han and B-L Liu contributed equally to this work)

\begin{abstract}
Background: $17 \alpha$-Hydroxylase/17,20-lyase deficiency (17OHD) caused by a mutation in the CYP17A1 gene is characterized by hypertension, hypokalemia, and abnormal development of the genitalia. The majority of CYP17A1 mutations are located in the coding sequence, and several intronic splicing site mutations have been reported.

Objective: A 2.5-year-old girl with 46,XY disordered sex development exhibited a nearly normal basal cortisol level and reduced sexual steroids. This study is aimed to explore the molecular basis and analyze its possible influence on the phenotype of the patient.

Methods and results: Mutation analysis revealed compound heterozygous CYP17A1 mutations, with c.985_987delinsAA in one allele and a synonymous substitution (c.1263G > A) in another allele. In vitro expression analysis of the allelic minigene showed that the novel nucleotide variation located in exon 8 induces a splicing signal, which results in an aberrant splicing of CYP17A1 mRNA and a missing portion of exon 8 . The translation product includes the deletion of six or seven amino acids from residue position 415 without causing a frameshift. Consistent with the result of molecular modeling, functional studies in transiently transfected HEK-293T cells with the aberrantly spliced enzyme proteins showed that the deleted proteins completely abolished the enzyme activity. However, RT-PCR indicated the existence of a small fraction of normal, functionally intact enzyme, which may explain the partial masculinization of this patient.

Conclusion: This is the first description of an exonic splicing mutation in CYP17A1 relevant to the $17 \mathrm{OHD}$ phenotype. It also demonstrates the importance of studying synonymous change in such patients with less severe phenotype.
\end{abstract}

European Journal of Endocrinology 164 627-633

\section{Introduction}

Human steroid 17 $\alpha$-hydroxylase/17,20-lyase (CYP17A1) catalyzes two distinct enzymatic steps in the steroidogenic pathway: $17 \alpha$-hydroxylation of pregnenolone or progesterone and 17,20-bond cleavage of the $17 \alpha-$ hydroxylated products to produce the $\mathrm{C} 19$ androgen precursors, mainly DHEA. As a branch point in the biosynthesis of cortisol and sex steroids, the enzyme is expressed in the adrenal glands and gonads. Adrenal $17 \alpha$-hydroxylase deficiency induces the elevation of ACTH and excessive production of mineralocorticoids, such as 11-deoxycorticosterone and corticosterone, resulting in low-renin hypertension and hypokalemia. The decreased production of sex steroids causes undervirilization of the external genitalia in 46 ,XY patients, leading to 46,XY disordered sex development (DSD), and an absence of pubertal development with primary amenorrhea in 46,XX patients $(1,2)$.

The CYP17A1 gene encoding this enzyme is mapped to chromosome 10q24.3 (3-5). To date, more than 50 deleterious mutations in the CYP17A1 gene have been reported to cause either combined or isolated $17 \alpha$ hydroxylase/17,20-lyase deficiency (17OHD), including missense mutations, deletions, insertions, and singlebase changes in the CYP17A1 gene. Mutations that interfere with steroid binding or heme binding mainly result in combined 17OHD, whereas mutations in the redox partner domain have been found to result in isolated 17,20-lyase deficiency $(1,6)$. In addition, several different intronic mutations disrupting splice sites have been reported (7-9).

In this study, we present a patient with ambiguous genitalia bearing a novel point mutation in exon 8 of the 
CYP17A1 gene. In this context, we demonstrate that this extronic mutation leads to aberrant splicing of the mRNA of CYP17A1 and provides evidence for the disease-causing effect of the newly identified mutation.

\section{Patients and methods}

\section{Subjects}

A girl from the Jiangsu province of China was first examined at the age of 2.5 years because of abnormal external genitalia. She was born in a non-consanguineous family in May 2006. Physical examination revealed a blood pressure of 90/50 $\mathrm{mmHg}$ (90th percentile for 2-year-old boy with height from 86 to $99 \mathrm{~cm}$ : 100-104/55-58 mmHg) (10), hypertrophic clitoris, blind-ending vagina, and an external urethral opening in the clitoris. Bilateral masses located in the inguinal canal were discovered. The karyotype was 46 ,XY. Ultrasonography revealed bilateral testis tissue measuring $18 \times 9 \mathrm{~mm}$ with an absence of the uterus and annexes. A commercial RIA kit (DiaSorin Ltd, Wokingham, UK) was used to measure the cortisol concentration. Testosterone, DHEA-S, 17-OHP, and ACTH concentrations were measured with an antibody-coated tube RIA kit (DSL-8600; Diagnostic Systems Laboratories, Inc., Webster, TX, USA). The serum $\mathrm{P}$ level was measured with the automated Abbott Architect i2000SR System (Abbott Laboratories). Plasma multisteroid analysis revealed a slightly reduced basal cortisol level $(187.6 \mathrm{nmol} / \mathrm{l}$ at $0800 \mathrm{~h}, 193.1-$ $606.9 \mathrm{nmol} / \mathrm{l})$, serum testosterone was $<0.28 \mathrm{nmol} / \mathrm{l}$ and DHEA-S $0.03 \mu \mathrm{mol} / \mathrm{l}$. The level of progesterone was $1.59 \mathrm{pmol} / \mathrm{l}(0.32-0.60 \mathrm{pmol} / \mathrm{l})$. Furthermore, aldosterone and 17-hydroxypregeterone were in the normal range, and the ACTH levels were also normal (12.0 pmol/l at $0800 \mathrm{~h}, 2.6 \sim 17.2 \mathrm{pmol} / \mathrm{l})$. The serum levels of $\mathrm{Na}, \mathrm{K}$, blood urea nitrogen, and creatinine were normal. Seven months later, she came to medical attention for a fever. Her temperature was $38^{\circ} \mathrm{C}$ and the total WBC count was $5.3 \times 10^{9} / \mathrm{l}$ with neutrophil levels at $40.2 \%$ and lymphocytes $57.2 \%$. The serum potassium and serum sodium levels were normal. After a brief anti-inflammatory therapy, ACTH stimulation test was performed. The basal ACTH level was remarkably increased (1049.5 pmol/l, $2.6 \sim 17.2 \mathrm{pmol} / \mathrm{l})$.
The measurement of basal and ACTH-stimulated steroids revealed elevated corticosterone and progesterone levels with the absence of a response of cortisol, which indicated the diagnosis of $17 \mathrm{OHD}$ (Table 1).

\section{CYP17A1 gene analysis}

After obtaining written informed consent, genomic DNA was extracted from peripheral blood leukocytes of the patient and her family members. All eight exons of the CYP17A1 gene were amplified by PCR using eight pairs of primers, as described previously (11). The PCR products were purified by using a gel extraction kit (Qiagen) and sequenced in both the sense and the antisense directions on an ABI 3700 DNA sequencer (Applied Biosystems PerkinElmer, Foster City, CA, USA). Once a mutation was identified, the corresponding PCR fragments obtained from the genomic DNA of 110 normal subjects (220 alleles) were also sequenced to exclude common polymorphisms.

\section{Functional studies}

Exons 5-8 of the mutant CYP17A1 gene were amplified using the father's genomic DNA with KOD-plus Polymerase (Toyobo, Osaka, Japan). The respective PCR products, consisting of $2543 \mathrm{bp}$, were amplified using the primers PC5F and PC8R (TCAGAGCTGCTTTCAGATAACCAC; TTGCCACAAGCTGAAAAAGAAG). The resulting products were ligated to the expression vector pcDNA3.1 (Invitrogen) between EcoRI and BamHI cloning sites (Fig. 1A). The wild-type and mutant minigene vectors were confirmed by direct DNA sequencing. Plasmids were isolated and purified by an anion exchange column (Qiagen). Plasmids $(4 \mu \mathrm{m})$ were transfected into HEK-293T cells by the lipofectamine method. RT-PCR amplification of cDNA was focused on the regions surrounding the exon 8 using the primers located in exons 7 and 8 of the CYP17A1 gene. The PCR products were electrophoresed on $20 \%$ PAGE. The different sized fragments were recovered from the gel and then sequenced with an ABI 3730 automated sequencer.

A deletion of 21nt (codons 415-421) was introduced into this construct (DEL7) using two rounds of PCR mutagenesis with the primers described below.

Table 1 Baseline steroid levels and responses to a $0.25 \mathrm{mg} \mathrm{ACTH}$ in the patient.

\begin{tabular}{lrrrc}
\hline Hormones $(\mathrm{nmol} / \mathrm{l})$ & \multicolumn{1}{c}{$\mathbf{0}^{\prime}$} & \multicolumn{1}{c}{$\mathbf{3 0}^{\prime}$} & \multicolumn{1}{c}{$\mathbf{6 0}^{\prime}$} & $\begin{array}{c}\text { Reference range } \\
\text { for basal levels }\end{array}$ \\
\hline Aldosterone & 1.31 & 3.20 & 3.69 & $0.11-0.87$ \\
Cortisol & 446.90 & 518.62 & 460.69 & $193.10-606.90$ \\
Corticosterone & 181.87 & 565.31 & 522.53 & $3.8-66.5$ \\
17OHP & 2.84 & 4.78 & 3.72 & $0.21-4.63$ \\
Progesterone (pmol/l) & 2.86 & 14.31 & 13.67 & $0.32-0.64$ \\
Testosterone & $<0.28$ & 0.59 & 0.52 & $6.24-29.12$ \\
\hline
\end{tabular}




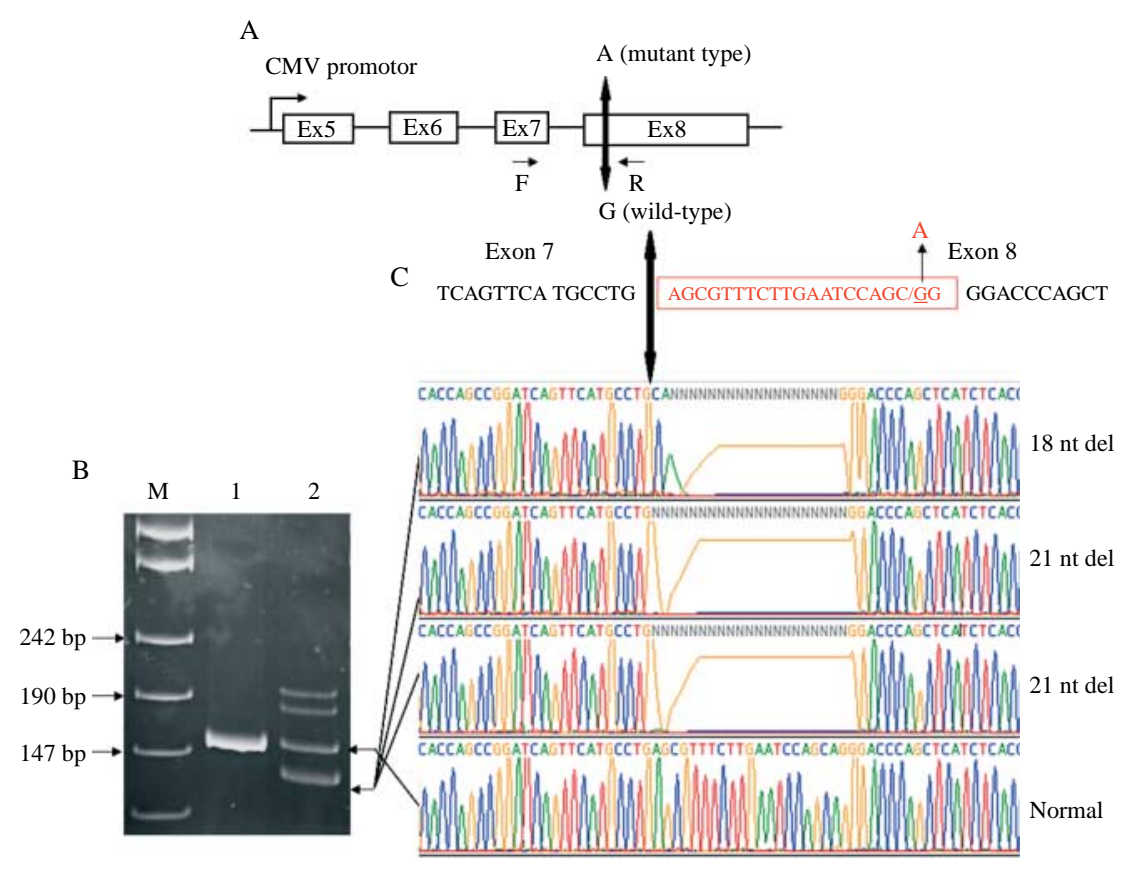

Figure 1 Effect of exonic mutation (c.1263G $>A$ ) on the variety of splice variants of the CYP17A1 gene. (A) Scheme of the mini-CYP17 gene expression vector construct. Exons are represented by boxes; introns are indicated by solid lines. The genomic fragments from exons 5 to 8 of the mutant and wild-type CYP17A1 genes were inserted in plasmid pcDNA3.1 to replace the plasmid sequence between the EcoRI and the BamHI sites. The arrows below exons 7 and 8 denote the positions of the primers used in RT-PCR. (B) The results of RT-PCR amplified from the HEK-293T cells transfected with the wild-type and mutant minigene vectors. M, marker; lane 1: HEK-293T cells transfected with wild-type plasmid. Lane 2: HEK-293T cells transfected with mutant plasmid. The sample of the HEK-293T cells transfected with the mutant minigene vector gave a variety of RT-PCR products (lane 3). (C) The sequencing results of the fragments amplified by RT-PCR from HEK-293T cells transfected with the wild-type and mutant plasmids. Totally, 18 or 21 nucleotides at the beginning of exon 8 were found to be deleted in the smaller $130 \mathrm{bp}$ fragment by sequencing. The deleted sequence is indicated in the red frame and the underlined alphabetical character indicates the site of the mutation.

The following primers were used with cloning sites underlined: CYP17 FOR: 5'-GGAATTCATGTGGGAGCTCGTGGCT-3' (EcoRI); CYP17 REV: 5'-CGGGATCCTTAGGTGCTACCCTCAGC-3' (BamHI); CYP17del21F: 5'-ATCAGTTCATGCCTGGGACCCAGCTCATCT-3'; CYP17del21R: 5'-AGATGAGCTGGGTCCCAGGCATGAACTGATCC-3'. Site-directed mutagenesis was used to create a mutant expression vector of 985_987delinsAA (329FS). The correction of the inserts was confirmed by sequencing, and the plasmids were purified with an anion exchange column (Qiagen).

HEK-293T cells were cultured in 24-well plates to $90 \%$ confluence and transfected with $0.8 \mu \mathrm{g}$ DNA per well using Lipofectamine 2000 (Invitrogen). Forty hours after transfection, various concentrations $(0.1,0.2,1.0$, $2.0 \mu \mathrm{mol} / \mathrm{l}$ ) of progesterone and 17-hydroxypregnenolone (Sigma) were added and incubated for another $6 \mathrm{~h}$. The medium was collected and frozen at $-20{ }^{\circ} \mathrm{C}$ until the assay of 17-hydroxyprogesterone (17 $\alpha$ hydroxylase activity) and DHEA (17,20-lyase activity) by RIA kits (Diagnostics Systems Laboratories) respectively. All values are expressed as mean \pm s.D. and represent the results of three independent experiments in duplicate.

\section{Molecular modeling}

To further approach the structural changes of the enzyme protein lacking the six or seven amino acids, we constructed a three-dimensional computer model with the NOC program (version 3.0; http://noch.sourceforge. net). Using the crystal structure of the human cytochrome P450 1A2 (PDB accession code 2HI4, chain A) as a template, CYP17A1 (residues 61-460) was modeled by homology with the SWISS-MODEL (http://swissmodel.expasy.org/) (12, 13). Mutations and deletions in CYP17A1 were modeled using the database search approach included in the Vector NTI Suite 9 (InforMax, Invitrogen).

\section{Results}

\section{CYP17A1 mutation analysis}

PCR direct sequencing revealed that one allele of the patient and her mother displayed a deletion-insertioncombined mutation (c.985_987delinsAA) at codon 329 exon 6 . A second mutation (c.1263G $>$ A) located on exon 8 was identified in another allele in the patient. 
This is a novel mutation, which was also detected in the father's sample. No additional mutation was found in the entire coding sequence or at the exon-intron boundaries. It is noteworthy that the novel mutation has no evident influence on the predicted amino acid coding sequence itself, as both triplets code for alanine. The PCR product of 110 normal subjects was sequenced, and no mutation was identified in this site of exon 8 , suggesting that the rearrangement is not a polymorphism.

\section{Functional splicing assay}

An in vitro expression study using a minigene system was performed to analyze the transcription of the patient's CYP17A1 gene (Fig. 1A). After the respective minigene was expressed in HEK-293T cells, the splicing site selection patterns for exons 5-8 were assessed based on the cDNA size corresponding to the expressed mRNA. The length of the PCR product was expected to be $151 \mathrm{bp}$ when the mRNA was collected from the cells transfected with the minigene, including the wildtype CYP17A1 gene. In comparison to the wild type, the splicing of intron 7 was influenced by the c.1263G $>$ A mutation of the CYP17A1 gene. PAGE revealed four fragments amplified from the cells transfected with the mutant vector, one of which was $151 \mathrm{bp}$, while one smaller product of approximately $130 \mathrm{bp}$ and two larger products were also present (Fig. 1B). The PCR products were later cloned into the pGEM-T vector, followed by sequencing of the plasmids.

After sequencing the selected 25 clones, we confirmed that these fragments contained at least three splicing products; in addition to the normal splicing product, two products were found to be missing $21 \mathrm{nt}$ or $18 \mathrm{nt}$ of the initiation of the exon 8 sequence of the CYP17A1 gene. Sequence determination of the splicing products suggested that the mutation c.1263G $>$ A constitutes a functional splice acceptor site, leading to a skipping of the 21 nt of the initiation of exon 8 in the mature mRNA of the CYP17A1 gene in vitro. At the same time, the AG at 1260 and 1261 were also activated, as a small portion of the transcripts was derived from splicing at this position, causing $\mathrm{c}$. [1244_1261Del; 1263 G>A] (Fig. 1C). However, the nucleotide skipping did not alter the reading frame of exon 8 but resulted in the deletion of six or seven amino acids from residue position 415 onward.

\section{Molecular modeling of CYP17A1}

We investigated the aberrant transcripts in a computerized CYP17A1 model based on the crystallized structure of cytochrome P450 1A2 in an effort to explain the putative effects of the mutations in the CYP17A1 molecule (Fig. 2A). The deleted amino acids Glu-Arg-Phe-Leu-Arg-Phe-Ala (415-421) are localized in the meander region between the $\mathrm{K}^{\prime}$-helix and the
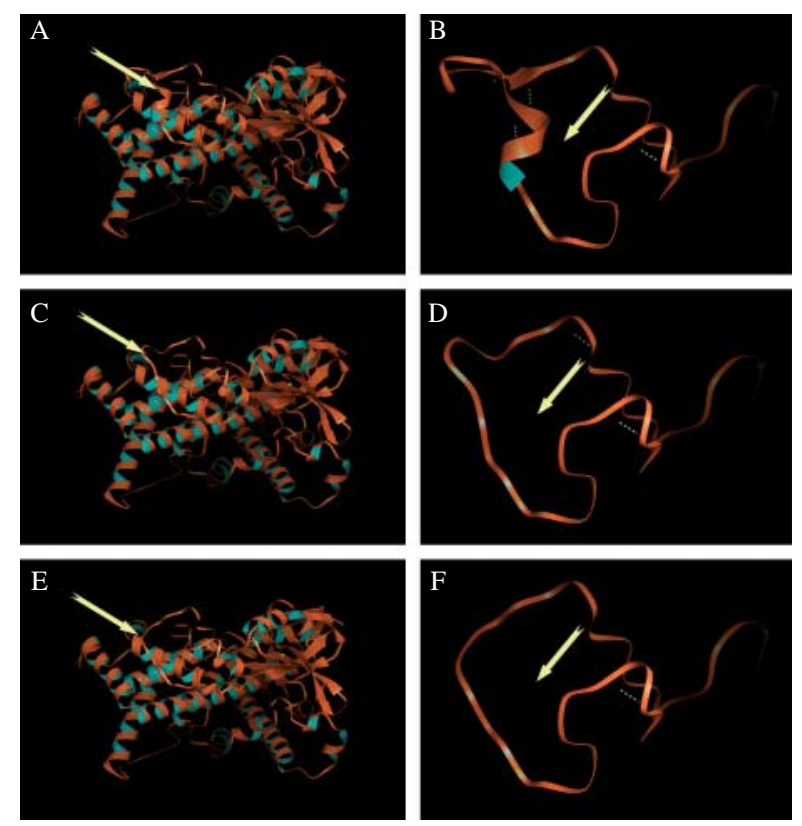

Figure 2 Molecular modeling of wild-type and mutant CYP17A1 with seven or six amino acids deleted in exon 8. The computer model is presented with a view vertical to the wild-type CYP17A1 protein $(A)$. The arrow indicates the helix structure in the meandering region of the enzyme. In CYP17-DEL7 and CYP17-DEL6, the helical structure in the meandering region was completely disrupted ( $\mathrm{C}$ and $\mathrm{E})$. Close-ups of the meandering region of CYP17A1 are shown in the right panel $(B, D$ and $F)$. In the regions CYP17-DEL7 and CYP17-DEL6, the main chain hydrogen bonds between Phe 417 and Ile426, Glu415 and Leu418, and Asn419 and GIn 424 were demolished. In the CYP17A-DEL 6, Ser427 formed a new hydrogen bond with Val430 (F).

heme-binding region (13). It is noteworthy that the Glu415-Arg 416-Phe417-Leu 418 region can form a regional helical structure. Furthermore, main chain hydrogen bonds are also formed between Phe 417 and Ile426, Glu415 and Leu418, and Asn419 and Gln 424 (Fig. 2B). When the amino acids located at residues 415-421 were absent, the meander region was shortened and the local helical structure disappeared (Fig. 2C and D). As a result, the main chain hydrogen bonds were obviously altered in the two types of mutant CYP17A1 (Fig. 2D and F). In CYP17A-DEL 6, Ser427 can form new hydrogen bond with Val430.

\section{In vitro functional assays}

To investigate whether the novel splicing variant of CYP17A1 with the amino acid 7 deletion possess any enzyme activity, we performed site-directed mutagenesis of the CYP17A1 cDNA in vitro. After transfecting CYP17A1 cDNA into HEK-293T cells for functional analysis, it was found that the enzyme produced by the wild-type CYP17A1 (WT) had full activity, which completely converted the substrate progesterone into $17 \alpha$-hydroxyprogesterone and converted the substrate 

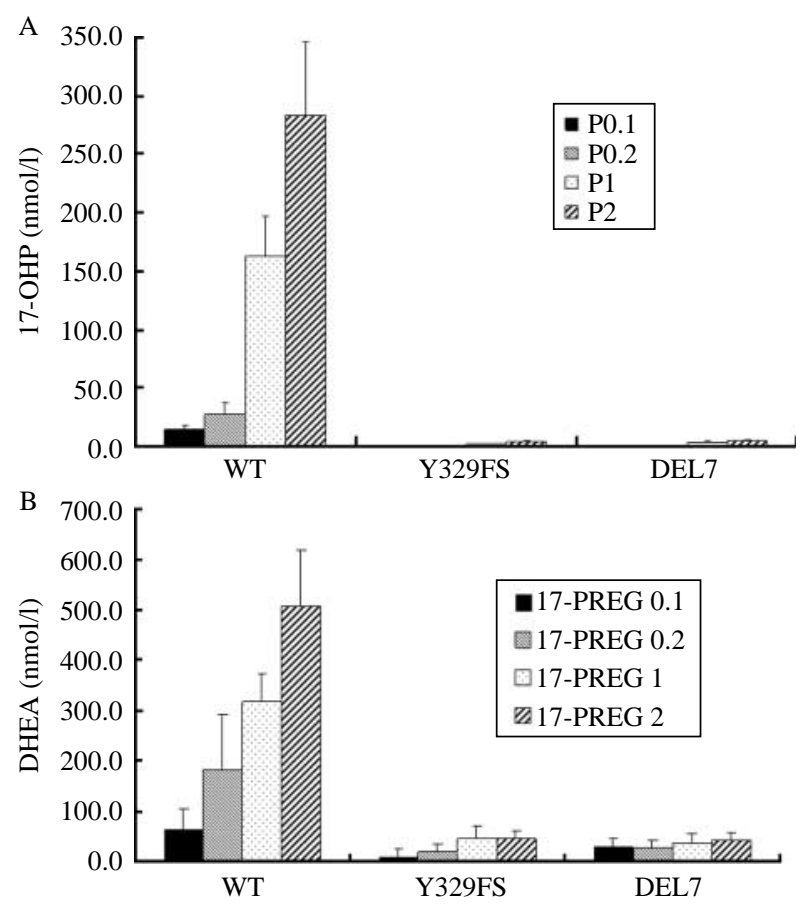

Figure 3 Enzymatic activity assay by in vitro expression. (A) The production of 17-hydroxyprogesterone (17-OHP) in the presence of various concentrations of progesterone $(P, 0.1,0.2,1.0$, and $2.0 \mu \mathrm{M}$ ) is shown as a measure of 17 -hydroxylase activity in HEK-293T cells transfected with wild-type, Y329FS, and DEL7 mutants. (B) The production of DHEA in the presence of various concentrations of 17-hydroxypregnenolone (17-PREG, 0.1, 0.2, 1.0 , and $2.0 \mu \mathrm{M}$ ) is shown as a measure of 17,20 -lyase activity in HEK-293T cells transfected with wild-type, Y329FS, and DEL7 mutants. Data are shown as mean \pm s.D. $(n=3$ in $A$ and B).

$17 \alpha$-hydroxypregnenolone into DHEA. The HEK-293 T cells expressing the DEL7 plasmid produced very low level of metabolites comparable to the positive control of HEK-293T cells containing 329FS. Even at the substrate concentrations of $2 \mu \mathrm{M}$, compared with WT, the conversion rates of progesterone to 17-hydroxyprogesterone and $17 \alpha$-hydroxypregnenolone to DHEA were nearly undetectable for 329FS and DEL7 mutated proteins (Fig. 3A and B). It showed that the protein produced by mutant cDNA with the deletion of codons 415-421 completely lost $17 \alpha$-hydroxylation activity and 17,20-lyase activity.

\section{Discussion}

$17 \mathrm{OHD}$ is not the rarest form of congenital adrenal hyperplasia $(\mathrm{CAH})$ in China. Almost 40 patients afflicted with $170 H D$ have been genotyped. The c.1517_1525del in exon 8 and the c.985_987delinsAA in exon 6 are reported to be the prevalent mutations of the CYP17A1 gene in China, nearly $90 \%$ of the patients bearing at least one of the mutations $(11,14-18)$. Both mutations were proved to completely abolish the CYP17A1 activity. Most affected individuals of either genetic sex present as phenotypic females with primary amenorrhea, sexual infantilism, hypertension, and hypokalemia owing to severely impaired enzymatic activity. In a few cases, patients manifested with partial $17 \alpha$-hydroxylase deficiency, exhibiting regular menses, intermittent hypertension, hypokalemia, and infertility. Genetic testing and in vitro transfection assays have demonstrated that the mutation of c.1418T $>C(F 453 S)$ and c.990_993del (E331del) retained part of the enzymatic activities, which is consistent with the phenotype of the patients $(14,17,19)$.

In this study, we report a complex heterozygous mutation of the CYP17A1 gene in our patient with 46,XY DSD. Apart from mutation of c.985_987delinsAA on exon 6 in one allele, an exonic splicing mutation (c.1263G $>$ A) induces certain aberrant splicing products with a deletion six or seven amino acids starting from residue position 415. The findings suggested that the splice site mutation does not completely impair the splicing, which is supported by the phenotype of our patient with 17OHD. Unlike patients genotyped as homozygotes for c.985_987delinsAA, this patient displayed a degree of masculinization of the external genitalia: clitoral enlargement and an external urethral opening in the clitoris. The basal hormone profile indicated nearly normal levels of cortisol and ACTH. However, under stress conditions, the levels of ACTH and corticosterone increased remarkably, while cortisol cannot be stimulated further. It is widely accepted that the degree of masculinization in the male fetus is related to residual activity of CYP17A1 and the magnitude of the consequent synthesis of fetal testosterone. And more than $25 \%$ of the normal enzymatic activity is necessary for normal fetal masculinization of the external genitalia (20). The mutation of c.985_987delinsAA has been

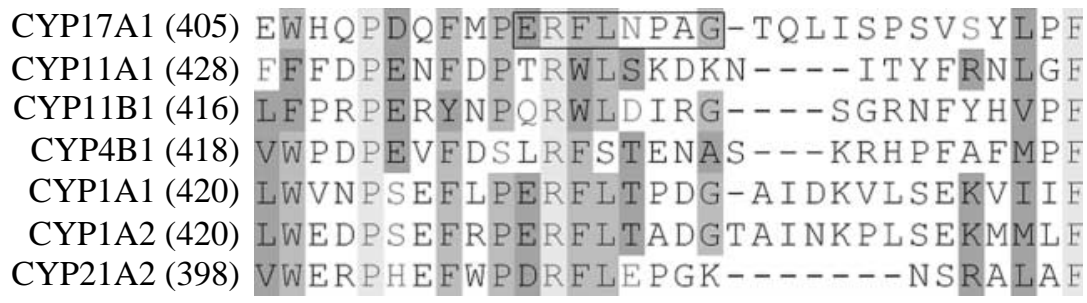

Figure 4 The amino acid Arg415-Phe418 is highly conserved through evolution. Partial amino acid sequences of human CYP17A1, around Arg416, are aligned with other related human P450s. The amino acids in the box represent the deleted sequences. The grey-shaded areas indicate the conserved amino acids in different degrees. 
proved to completely abolish the activity of CYP17A1. Therefore, the residual $17 \alpha$-hydroxylase and 17,20lyase activity were derived from the wild-type transcripts induced by the exonic splicing mutation of another allele. It was postulated that the residual enzyme activity of the normal transcripts in the patient was $<25 \%$.

Analysis of the $17 \alpha$-hydroxylase and 17,20-lyase activity of the deleted protein revealed an absence of enzymatic activity, comparable to the mutant enzyme of c.985_987delinsAA. In fact, Arg416His and Arg416Cys mutations of CYP17A1 gene have been reported to induce a complete lack of the enzymatic activity, demonstrating the critical role of Arg416 (14, 19). From the molecular model of CYP17A1, the amino acids Glu415-Arg416 were located in the C-terminal of the meandering region between the $\mathrm{K}^{\prime}$-helix and the hemebinding region, well upstream from the crucial Cys442. The meandering region of CYP17A1, consisting of ten amino acids stretched from His 407 to Arg 416, is a typical structural domain of $\mathrm{P} 450$ that appears to participate in binding the redox partner and $\mathrm{P} 450$ oxidoreductase $(13,15,21)$. From the protein sequence alignments, it was demonstrated that Glu415, Arg416, Phe417, and Leu418 are conserved in other human cytochrome P450 proteins (CYP21A2, CYP11A1, CYP11B1, CYP1A1, CYP1A2, and CYP4B1) (Fig. 4). The local loop structure composed of Glu415-Arg 416Phe417-Leu 418 in the meandering region was abolished when the amino acids were deleted. The shortening of the meandering region results in deprivation of main chain hydrogen bond interactions as well as a side chain, leading to a disorientation of the heme group relative to the protein.

The age of onset of overt hypertension and hypokalemia may significantly vary, even in patients with the same genotype $(2,22)$. In contrast with the majority of cases who were diagnosed after puberty, our patient did not exhibit hypertension and hypokalemia, despite the increased levels of corticosterone and aldosterone under stress. It may be associated with the response of immature renal tubular to the elevated mineralocorticoids. Normal even elevated levels of aldosterone have long been a controversial phenomenon. Various assay methods, dietary sodium intake, and differing severity of the enzymatic defect further complicate the problem $(1,22,23)$. However, Dhir et al. (22) have pointed out that the degree of hypertension seems to be more closely related to the time span without treatment than to the CYP17A1 genotype. So careful examination and followup might be helpful toward improving the prognosis of the patient.

\section{Declaration of interest}

The authors declare that there is no conflict of interest that could be perceived as prejudicing the impartiality of the research reported.

\section{Funding}

This work was supported by a grant from the National Natural Science Foundation Program (81070666), People's Republic of China. We are very grateful to Prof. Richard J Auchus for his assistance in plasmid construction. Pacific Edit reviewed the manuscript prior to submission.

\section{References}

1 Auchus RJ. The genetics, pathophysiology, and management of human deficiencies of P450c17. Endocrinology and Metabolism Clinics of North America 200130 101-119. (doi:10.1016/S08898529(08)70021-5)

2 Yanase T, Simpson ER \& Waterman MR. 17-Hydroxylase/17,20lyase deficiency: from clinical investigation to molecular definition. Endocrine Reviews 199112 91-108. (doi:10.1210/edrv-12-1-91)

3 Matteson KJ, Picado-Leonard J, Chung BC, Mohandas TK \& Miller WL. Assignment of the gene for adrenal P450c17 (steroid 17 alphahydroxylase/17,20-lyase) to human chromosome. Journal of Clinical Endocrinology and Metabolism 198663 789-791. (doi:10. 1210/jcem-63-3-789)

4 Fan YS, Sasi R, Lee C, Winter JS, Waterman MR \& Lin CC. Localization of the human CYP17 gene (cytochrome P450(17alpha)) to 10q24.3 by fluorescence in situ hybridization and simultaneous chromosome banding. Genomics 199214 1110-1111. (doi:10.1016/S0888-7543(05)80140-5)

5 Picado-Leonard J \& Miller WL. Cloning and sequence of the human 17 gene for P450c17 (steroid 17 alpha-hydroxylase/17,20 lyase): similarity with the gene for P450c21. DNA 19876 439-448. (doi:10.1089/dna.1987.6.439)

6 Tiosano D, Knopf C, Koren I, Levanon N, Hartmann MF, Hochberg Z \& Wudy SA. Metabolic evidence for impaired 17alpha-hydroxylase activity in a kindred bearing the E305G mutation for isolate 17,20-lyase activity. European Journal of Endocrinology 2008158 385-392. (doi:10.1530/EJE-07-0712)

7 Yamaguchi H, Nakazato M, Miyazato M, Toshimori H, Oki S, Shimizu K, Suiko M, Kangawa K \& Matsukura S. Identification of a novel splicing mutation and 1-bp deletion in the 17alphahydroxylase gene of Japanese patients with 17alpha-hydroxylase deficiency. Human Genetics 1998102 635-639. (doi:10.1007/ s004390050754)

8 Yamaguchi H, Nakazato M, Miyazato M, Kangawa $\mathrm{K}$ \& Matsukura S. A $5^{\prime}$-splice site mutation in the cytochrome P450 steroid 17alpha-hydroxylase gene in 17alpha-hydroxylase deficiency. Journal of Clinical Endocrinology and Metabolism 199782 1934-1938. (doi:10.1210/jc.82.6.1934)

9 Costa-Santos M, Kater CE, Dias EP \& Auchus RJ. Two intronic mutations cause 17-hydroxylase deficiency by disrupting splice acceptor sites: direct demonstration of aberrant splicing and absent enzyme activity by expression of the entire CYP17 gene in HEK-293 cells. Journal of Clinical Endocrinology and Metabolism 200489 43-48. (doi:10.1210/jc.2003-031020)

10 Rosner B, Cook N, Portman R, Daniels S \& Falkner B. Determination of blood pressure percentiles in normal-weight children: some methodological issues. American Journal of Epidemiology 2008 167 653-666. (doi:10.1093/aje/kwm348)

11 Qiao J, Hu RM, Peng YD, Song HD, Peng YW, Gao GF, Hao JH, Hu NY, Xu MY \& Chen JL. A complex heterozygous mutation of His373Leu and Asp487-Ser488-Phe489 deletion in human cytochrome P450c17 causes 17alpha-hydroxylase/17,20-lyase deficiency in three Chinese sisters. Molecular and Cellular Endocrinology 2003201 189-195. (doi:10.1016/S0303-7207 (02)00156-9)

12 Arnold K, Bordoli L, Kopp J \& Schwede T. The SWISS-MODEL Workspace: a web-based environment for protein structure homology modelling. Bioinformatics 200622 195-201. (doi:10. 1093/bioinformatics/bti770) 
13 Auchus RJ \& Miller WL. Molecular modeling of human P450c17 (17alpha-hydroxylase/17,20-lyase): insights into reaction mechanisms and effects of mutations. Molecular Endocrinology 199913 1169-1182. (doi:10.1210/me.13.7.1169)

14 Yang J, Cui B, Sun S, Shi T, Zheng S, Bi Y, Liu J, Zhao Y, Chen J, Ning G \& Li X. Phenotype-genotype correlation in eight Chinese 17alpha-hydroxylase/17,20lyase-deficiency patients with five novel mutations of CYP17A1 gene. Journal of Clinical Endocrinology and Metabolism 200691 3619-3625. (doi:10.1210/jc.2005-2283)

15 Lam CW, Arlt W, Chan CK, Honour JW, Lin CJ, Tong SF, Choy KW \& Miller WL. Mutation of proline 409 to arginine in the meander region of cytochrome p450c17 causes severe 17 alpha-hydroxylase deficiency. Molecular Genetics and Metabolism 200172 254-259. (doi:10.1006/mgme.2000.3134)

16 Wei JQ, Wei JL, Li WC, Bi YS \& Wei FC. Genotyping of five chinese patients with 17alpha-hydroxylase deficiency diagnosed through high-performance liquid chromatography serum adrenal profile: identification of two novel CYP17 mutations. Journal of Clinical Endocrinology and Metabolism 200691 3647-3653. (doi:10. 1210/jc.2006-0153)

17 Tian Q, Zhang Y \& Lu Z. Partial 17alpha-hydroxylase/17,20-lyase deficiency-clinical report of five Chinese $46, \mathrm{XX}$ cases. Gynecological Endocrinology 200824 362-367. (doi:10.1080/0951359080 2194051)

18 Qiao J, Chen X, Zuo CL, Gu YY, Liu BL, Liang J, Lu YL, Tang JF, Wu YX, Chen MD, Chen JL, Wu WL \& Song HD. Identification of steroid biosynthetic defects in genotype-proven heterozygous individuals for 17alpha-hydroxylase/17,20-lyase deficiency. Clinical Endocrinology 200972 312-319. (doi:10.1111/j.13652265.2009.03607.x)

19 Rosa S, Duff C, Meyer M, Lang-Muritano M, Balercia G, Boscaro M, Topaloglu AK, Mioni R, Fallo F, Zuliani L,
Mantero F, Schoenle EJ \& Biason-Lauber A. P450c17 deficiency: clinical and molecular characterization of six patients. Journal of Clinical Endocrinology and Metabolism 200792 1000-1007. (doi:10.1210/jc.2006-1486)

20 Miura K, Yasuda K, Yanase T, Yamakita N, Sasano H, Nawata H, Inoue M, Fukaya T \& Shizuta Y. Mutation of cytochrome P-45017 alpha gene (CYP17) in a Japanese patient previously reported as having glucocorticoid-responsive hyperaldosteronism: with a review of Japanese patients with mutations of CYP17. Journal of Clinical Endocrinology and Metabolism $1996 \mathbf{8 1}$ 3797-3801. (doi:10.1210/jc.81.10.3797)

21 Pikuleva IA, Cao C \& Waterman MR. An additional electrostatic interaction between adrenodoxin and P450c27 (CYP27A1) results in tighter binding than between adrenodoxin and p450scc (CYP11A1). Journal of Biological Chemistry 199922 2045-2052. (doi:10.1074/jbc.274.4.2045)

22 Dhir V, Reisch N, Bleicken CM, Lebl J, Kamrath C, Schwarz HP, Grötzinger J, Sippell WG, Riepe FG, Arlt W \& Krone N. Steroid 17alpha-hydroxylase deficiency: functional characterization of four mutations (A174E, V178D, R440C, L465P) in the CYP17A1 gene. Journal of Clinical Endocrinology and Metabolism $2009 \mathbf{9 4}$ 3058-3064. (doi:10.1210/jc.2009-0172)

23 Turan S, Bereket A, Guran T, Akcay T, Papari-Zareei M \& Auchus RJ. Puberty in a case with novel 17-hydroxylase mutation and the putative role of estrogen in development of pubic hair. European Journal of Endocrinology 2009160 325-330. (doi:10. 1530/EJE-08-0632)

Received 10 January 2011

Accepted 31 January 2011 